\title{
AVALIAÇÃO ULTRASSONOGRAFIA DA ECOGENICIDADE DO PARÊNQUIMA TESTICULAR COMO EXAME COMPLEMENTAR NO ANDROLÓGICO DE CARNEIROS
}

\author{
TEIXEIRA, Pedro Paulo Maia ${ }^{1}$ \\ OLIVEIRA, Maria Emilia Franco ${ }^{2}$ \\ D'AMATO, Carla Cristina ${ }^{3}$ \\ RODRIGUES, Luiz Fernando de Souza ${ }^{4}$ \\ VICENTE, Wilter Ricardo Russiano ${ }^{3}$
}

RESUMO: O objetivo deste trabalho foi padronizar um método de ultrassonografia para exames do parênquima testicular em andrológicos de carneiros $(n=6)$. Foi realizada biometria testicular, espermiograma e exame ultrassonográfico do parênquima testicular, realizando imagens do parênquima testicular e aferido o percentual de ecogenicidade em pela escala de cinza no próprio aparelho, correlacionando com dados da biometria e espermiograma. Verificaram-se correlações positivas entre a ecogenicidade e a concentração espermática $\left(p=0,0192\right.$ e $\left.r^{2}=0,4373\right)$ e entre ecogenicidade e motilidade massal $\left(p=0,0358\right.$ e $\left.r^{2}=0,37\right)$, demonstrando que o método é eficiente para diagnosticar alterações escrotais assintomáticas aos exames convencionais, contribuindo para a reprodução animal.

Palavras-chave: Ultrassom. Parênquima testicular. Carneiros. Exame andrológicos.

\section{ECHOGENECITY EVALUATION OF THE TESTICULAR PARENCHYMA AND CORRELATION WITH THE PHYSICAL CHARACTERISTICS OF SEMEN IN RAMS}

SUMMARY: The present study aimed to standardize an ultrasonogram method to testicular parenchyma in andrological sheep $(\mathrm{n}=6)$. We made testicular biometric, spermiogram and testicular parenchyma ultrasonography, carrying images of the testicular parenchyma and measured the percentage of the echogenicity in gray scale on the device itself, correlating with the biometrics data and spermiogram. There were positive correlations between echogenicity and sperm concentration $(\mathrm{p}=0.0192$ and $\mathrm{r} 2=0.4373)$ and between echogenicity and mass motility $(\mathrm{p}=0.0358$ and $\mathrm{r} 2=0.37)$, demonstrating that the method is efficient to detect abnormal scrotal asymptomatic by conventional tests, contributing to animal breeding.

Keywords: Ultrasound. Testicular parenchyma. Rams. Andrological examination.

\section{INTRODUÇÃO}

A ovinocultura vem apresentando crescimento no país e no mundo (FONSECA, 2005), neste contexto os reprodutores são de fundamental importância na produtividade final do sistema. Tanto em monta natural quanto em programas de inseminação artificial ou outras

\footnotetext{
${ }^{1}$ Aprimoramento em Reprodução de caprinos e ovinos; Mestre em Cirurgia Veterinária - FCAV/UNESP.

${ }^{2}$ Mestre em Medicina Veterinária pela Universidade Estadual Paulista Júlio de Mesquita Filho

${ }^{3}$ FCAV/UNESP

${ }^{4}$ CPCOP/ISPA/UFRA
} 
biotécnicas de reprodução, os machos os principais responsáveis pelo melhoramento genético do rebanho por ser passível de maior pressão de seleção (SIMPLICIO et al., 2007). Assim, a escolha de um reprodutor deve estar baseada em sua produção, avaliando-se o teste de progênie, e também através da realização de um exame clínico-andrológico minucioso (MARTINS, 2006).

O uso de reprodutores testados e com alta capacidade fertilizante é de grande importância para garantir boa eficiência reprodutiva e produção de cordeiros. Desse modo, a busca por indicadores da fertilidade de reprodutores tem sido o alvo de diversos estudos nos últimos anos. Smith et al. (1989) indicam que o potencial reprodutivo animal não pode ser expresso pela análise de apenas um indicador, devendo este ser estimado através da combinação de vários parâmetros.

A capacidade reprodutiva dos reprodutores é avaliada, com precisão, pelo exame andrológico (UNANIAN, 2000). Porém se fez necessário a evolução das técnicas de diagnóstico, sendo a ultrassonografia um método muito importante (SANCHES; AFONSO, 2000; VIANA, 2004), podendo avaliar a anatomia das glândulas sexuais e o parênquima testicular dos reprodutores, podendo diagnosticar alterações andrológicas ainda assintomáticas aos exames convencionais (JUCÁ et al., 2009).

No entanto em carneiros ainda não foi estabelecida avaliação precisa e não subjetiva, não havendo um método que possa analisar a ecogenicidade de órgãos do sistema reprodutor através da sua escala de cinza (PASTORE, 2008; CARDILLI et al., 2009)

Com esse intuito este estudo objetivou avaliar por ultrassonografia a ecogenicidade do parênquima testicular em ovinos adultos, em fase reprodutiva, correlacionado os achados com o exame andrológico (biometria e espermiograma).

\section{MATERIAL E MÉTODOS}

Foram avaliados 6 animais adultos em idade reprodutiva, da raça Dorper e Santa Inês de propriedades mantidos em uma central de andrologia no Município de Jaboticabal (São Paulo, Brasil, latitude $21^{\circ} 15^{\prime} 17^{\prime \prime}$ sul e longitude 48 $19^{\prime} 20^{\prime \prime}$ oeste, estando a uma altitude de 605 metros e predominância climática do tipo CFa segundo a classificação de Köppen).

Estes foram submetidos exames ultrassonográficos do parênquima testicular, mensuração da biometria testicular e avaliação do sêmen.

Foi utilizado um aparelho da marca Pie Medical, e modelo Áquila, ligado ao transdutor linear $6 \mathrm{MHz}$. Os exames ultrassonográficos foram executados no parênquima testicular, utilizando-se gel acústico sobre a bolsa testicular previamente tricotomizada nas 
regiões aferição. Foram feitas imagens em planos sagitais, transversais e frontais nos testículos direito e esquerdo.

Com a função de aferição de ecogenicidade (escala de cinza) da imagem, no próprio aparelho foram selecionadas três áreas predeterminadas do parênquima tido o cuidado para que não sejam aferidas imagens das túnicas, pele, epidídimo e o mediastino, englobando desta forma somente o parênquima testicular.

Realizou-se a inspeção clínica das estruturas externas do sistema reprodutor (simetria e forma dos testículos e epidídimos e estruturas do plexo panpiniforme). Com o auxílio de paquímetro e fita métrica foi obtidas a circunferência escrotal, comprimento, largura, espessura dos testículos.

Para determinar a forma dos testículos (forma do testículo esquerdo - FTe, forma do testículo direito - FTd e média das formas dos testículos - FTm), se calculou a razão entre a sua largura e o comprimento (razão LARG/COMP), segundo BAILEY et al. (1996), na escala de 1 a 0,5, em que 1 significa $L A R G=$ COMP e 0,5, LARG = 1/2 COMP. Em função desta escala, foram estabelecidas as seguintes formas: razão $\mathrm{I} \leq 0,5=$ longo; razão II de 0,51 a 0,625 = longo/moderado; razão III de 0,626 a 0,750 = longo/oval; razão IV de 0,751 a $0,875=$ oval/esférico; e razão $\mathrm{V}>0,875$ = esférico.

O volume foi mensurado compararam-se duas equações matemáticas para estimar o volume testicular. Uma através do modelo matemático sugerido por FIELDS et al., (1979) e UNANIAN et al.,(2000) com a equação matemática do volume do cilindro: VOLC $=2 \mathrm{x}$ [(LARG/2) $\left.)^{2} \times \Pi \times(\mathrm{COMP})\right], \pi=3,141592654$, e outra pelo sugerido por BAILEY et al. (1998), a equação do prolato esferóide VOLP $=2 \times\left[4 / 3 \times \Pi \times(\mathrm{LARG} / 2)^{2} \times(\mathrm{COMP} / 2)\right]$, também se fez a média do testículo direito e esquerdo (VOLm) e a somatória considerando o

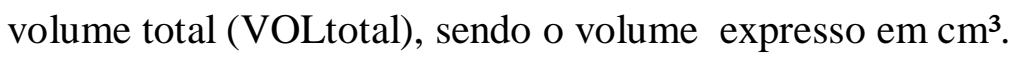

Para análise de sêmen, a coleta foi realizada através do método da vagina artificial, segundo Mies Filho (1980). O espermiograma foi realizado segundo CBRA (1998), avaliando o volume do ejaculado, turbilhonamendo, motilidade, vigor, concentração espermática total e concentração espermática por mL. Além do número de patologias espermáticas.

Os dados foram expressos em média e desvio padrão, realizando o teste one-way ANOVA (não paramétricos) e regressão linear para correlação entre os dados de ecogenicidade, biometria e espermiograma, empregado o pacote estatístico Grafped Prisma 4.

\section{RESULTADOS E DISCUSSÃO}

Os resultados da biometria testicular foram de expressos na tabela 1 e a análise de sêmen foi expressa na tabela 2 . 
Tabela 1. Parâmetros biométricos

\begin{tabular}{cc}
\hline CE & $36,75 \pm 4,1$ \\
COMPd & $9,883 \pm 1,4$ \\
COMPe & $9,583 \pm 1,0$ \\
LARGd & $6,3 \pm 0,3$ \\
LARGe & $6,6 \pm 0,8$ \\
FTd & $0,659 \pm 0,125$ \\
FTe & $0,691 \pm 0,078$ \\
VOLCd & $636,2 \pm 164,4$ \\
VOLCe & $674,4 \pm 236,3$ \\
VOLPd & $424,1 \pm 109,6$ \\
VOLPe & $449,6 \pm 157,5$
\end{tabular}

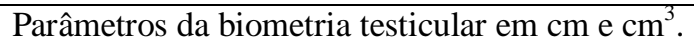

Tabela 2. Parâmetros seminais entre o período seco e chuvoso.

\begin{tabular}{cc}
\hline Volume & $1,852 \pm 0,6$ \\
Turbilhonamento & $4,81 \pm 0,2$ \\
Concentração $\left(10^{9} \mathrm{sptz} / \mathrm{ml}\right)$ & $4,5 \pm 9,4$ \\
Motilidade & $81,09 \pm 5,917$ \\
Vigor & $4,76 \pm 0,40$ \\
Defeitos totais & $8,36 \pm 6,93$ \\
\hline
\end{tabular}

A forma dos testículos apresentaram forma longa/oval apresentando 0,659 $\pm 0,125 \mathrm{e}$ 0,691 \pm 0,0782 para o testículo direito e esquerdo respectivamente, como observado por LOUVANDINI et al. (2008). O VOLC apresentou-se estatisticamente igual ao VOLP ( $\mathrm{P}>0,05$ ), sendo a média deste sendo $714,6 \pm 209,6 \mathrm{~cm}^{3}$ e a soma dos volumes dos dois testículos $1429 \pm 419,2 \mathrm{~cm}^{3}$.

O Parênquima testicular apresentou-se no ultrassom, de uma forma geral, homogêneo, com ecogenicidade $37,91 \pm 5,2 \%$. Verificou-se que não houve diferença entre a ecogenicidade entre os planos aferidos $(p>0,05)$, o que já era esperado pela homogeneidade do parênquima testicular. Também se verificou uma correlação entre a ecogenicidade com o turbilhonamento do sêmen $\left(\mathrm{P}=0,0358, \mathrm{r}^{2}=0,37\right)$, e da ecogenicidade com a concentração espermática $\left(\mathrm{P}=0,0192, \mathrm{r}^{2}=0,43\right)$, sendo uma correlação moderada, podendo ser influenciada pelo baixo número de animais (Fig.2). Verificou-se que um dos animais apresentou ecogenicidade $32 \pm 7,763 \%$, abaixo da média do grupo, apresentando pontos hiperecóicos e 
regiões hipoecóicas no parênquima testicular, manifestando na qualidade seminal inferior aos demais. Sendo este apresentado $22 \%$ de defeitos espermáticos totais resultado muito mais elevado que a média do grupo $(5,15 \pm 6,939 \%)$.

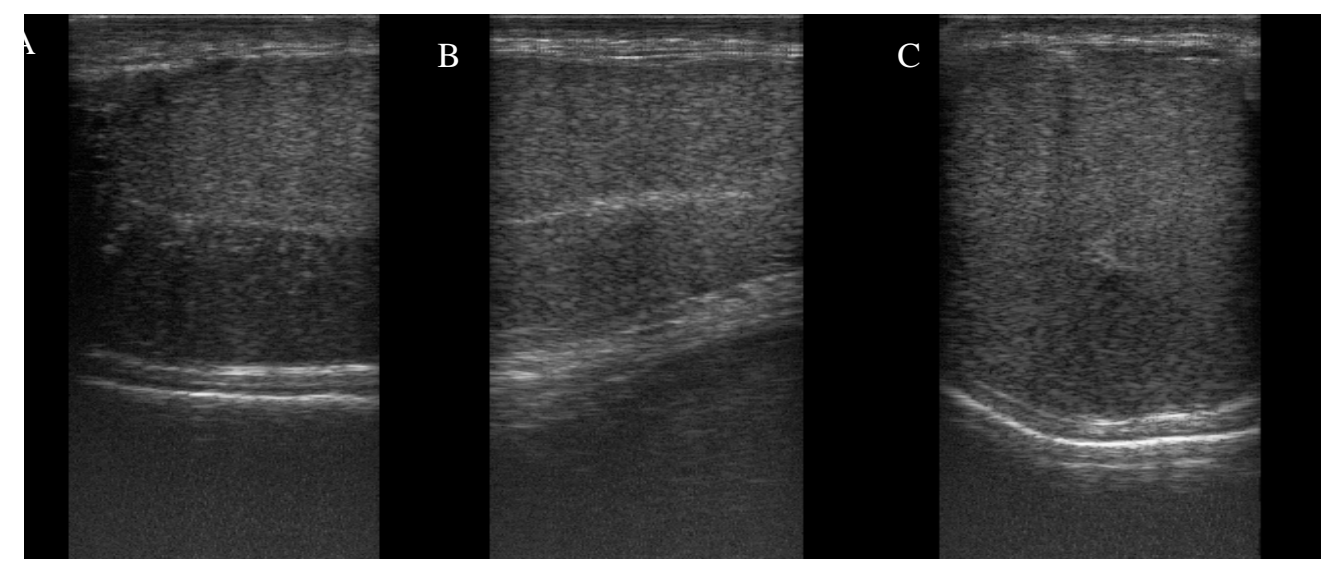

Figura 1: Imagens utrassonogáficas do parênquima testicular, "A" corte sagital, "B" corte frotal e "C" corte transversal.
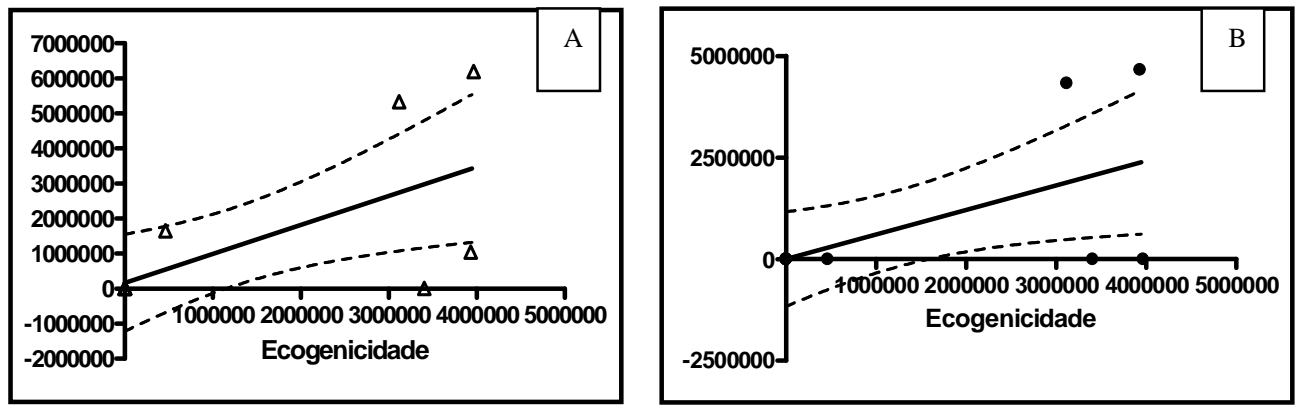

Figura 2: Gráfico da regressão linear, "A" ecogenicidade x concentração espermática e "B" ecogenicidade x turbilhonamento.

\section{CONCLUSÃO}

O estudo demonstrou que a metodologia empregada apresentou grande contribuição para os achados andrológicos, mostrando com precisão e de maneira prática a ecogenicidade do parênquima testicular. Mesmo tendo ocorrido somente uma correlação moderada, teve influência em alguns parâmetros seminais, contribuindo para complementação do exame andrológico, auxiliando no diagnostico precoce de diversas alterações reprodutivas do macho.

\section{REFERÊNCIAS}

CARDILLI, D.J.et al. Alterações do padrão ultra-sonográfico do parênquima testicular em bovinos jovens da raça Nelore. In: CONGRESSO BRASILEIRO DE REPRODUÇÃO ANIMAL, 18, Belo Horizonte, MG. Anais ... Belo Horizonte: CBRA, 2009. (CD-ROM). ISSN1984-871. Pag.269, 2009. 
FIELDS, M. J. Age, season and breed effects on testicular volume and semen traits in young beef bulls. J Anim Sci, v.48, p. 1299 - 1304, 1979.

FONSECA J.F. Estratégias para o controle do ciclo estral e superovulação em ovinos e caprinos. In: CONGRESSO BRASILEIRO DE REPRODUÇÃO ANIMAL, 16, 2005, Anais... CBRA. 2005.

JUCÁ, A.F.et al. Avaliação ultrassonográfica dos testículos e das glândulas sexuais anexas de carneiros Santa Inês. Ciência Animal Brasileira, v. 10, n. 2, p. 650-659, abr./jun. 2009.

LOUVANDINI, H.et al. Características biométricas testiculares em carneiros Santa Inês submetidos a diferentes regimes de suplementação protéica e tratamentos anti-helmínticos Ciên Anim Bras, v. 9, n. 3, p. 638-647, jul./set. 2008.

MARTINS, L.F.; PEREIRA, M.C.B.; GUIMARAES, J.D. et al. Avaliação espermática e da concentração de proteínas solúveis no plasma seminal de bodes da raça Alpina em regime de monta controlada. Rev Bras Zoo, v.35, n.4, p.1653-1659, 2006.

MIES FILHO, A.; PUGA, J.M.P.; JOBIM, M.I.M. et al.. Biometria testicular em bovino. I Relação entre idade e medidas testiculares. Rev Bras Repro Anim, v.4, p. 56-65, 1980.

PASTORE, A.A. Ultra-sonografia na avaliação andrológica de bovinos da raça nelore. 2008. 81 f. 2008. Tese (Doutorado em Reprodução Animal.) - FCAV/UNESP, Jaboticabal.

SÁNCHEZ, R., ALFONSO, E. Ultra-sonografia na reprodução animal. TecnoVet, v.1, p.6, 2000.

SÁNCHEZ, R., ALFONSO, E. Ultra-sonografia na reprodução animal. TecnoVet, v.1, p.6, 2000.

SIMPLÍCIO A.A.V., FREITAS V.J.F., FONSECA J.F. Biotechniques of reproduction as techniques of reproductive management in sheep. Rev Bras Repro Anim, v.31, n.2, p. 234246, 2007.

SMITH, M.F.; BRINKS, J.S.; RICHARDSON, G.V. Estimation of gentetic parameters among soundness examination components and growth traits in yearling bulls. J Anim Scie. v.67, p.2892 - 2896, 1989.

UNANIAN, M.M.et al. Características biométricas testiculares para avaliação de touros zebuínos da raça Nelore. Rev Bras Zoot, v. 29, n. 1, p. 136-144, 2000.

VIANA, J.H.M.; BARRETO FILHO, J.B.; ALBUQUERQUER, F.T. Ultra-som: ferramenta para melhorar a eficiência reprodutiva. Lavras: UFLA, 2004. 